Check for updates

Cite this: RSC Adv., 2019, 9, 35328

Received 26th September 2019 Accepted 21st October 2019

DOI: $10.1039 / c 9 r a 07826 f$

rsc.li/rsc-advances

\section{Fabrication of centrifugally spun prepared poly(lactic acid)/gelatin/ciprofloxacin nanofibers for antimicrobial wound dressing}

\begin{abstract}
Lei Xia, ${ }^{a}$ Linlin Lu, (D) ${ }^{a}$ Yuxia Liang ${ }^{\mathrm{b}}$ and Bowen Cheng (D) *ac
Centrifugal spinning is a novel technology for producing ultrafine fibers in high yield with diameters ranging from micro to nanometers. The obtained fibers have potential applications in the field of tissue engineering, wound dressing, and biomedicine etc. In this paper, a system of poly(lactic acid)/gelatin (PLA/GE) nanofibers loaded with ciprofloxacin (CPF) drug for wound dressings were successfully prepared by centrifugal spinning. The nanofibers were characterized by scanning electron microscopy (SEM) coupled with energy dispersive spectroscopy (EDS), X-ray diffraction (XRD), thermal gravimetric analysis (TGA) and Fourier transform infrared spectroscopy (FTIR). In addition, the nanofibers' properties in terms of hydrophilicity, antibacterial properties and in vitro drug release were further investigated. The results showed that the CPF drug was successfully loaded and in an amorphous state in the PLA/GE nanofibers, the surface of the nanofibers was smooth and the nanofibers' diameter became large after the drug was loaded. The thermal stability of the nanofiber was reduced while the hydrophilicity was improved. Antibacterial and in vitro drug release experiments showed that the nanofibers have obvious antibacterial properties and have the positive effect of sustained release of the drug. Drug-loaded PLA/GE nanofibers could be good candidates for wound dressing.
\end{abstract}

\section{Introduction}

Centrifugal spinning, a newly developed method for generating micro to nanofibers, has unique advantages including low cost, rapid production, wide materials choice, highly aligned nanofibers, and is independent of the electric field when compared with electrospinning. ${ }^{1-3}$ During the centrifugal spinning process, the spinning solution is ejected from the needle to form a jet when the centrifugal force generated by the direct current motor is greater than a critical value determined by the balance between the surface tension of the liquid and the centrifugal force. The jet then undergoes a stretching process, accompanied by rapid evaporation of the solvent, and is eventually deposited on the collectors forming dried nanofibers. ${ }^{4-6}$ Since the centrifugal spinning process does not use a highvoltage electric field, it alleviates the related safety concern. In this process, the high rotational speed allows fast and scalable fiber fabrication, which can dramatically improve the production rate by two to three orders of magnitude and reduce the production cost when compared with the electrospinning

${ }^{a}$ School of Textile Science and Engineering, State Key Laboratory of Separation Membranes and Membrane Processes/National Center for International Joint Research on Separation Membranes, Tianjin Polytechnic University, Tianjin 300387, China.E-mail: bowen15@tjpu.edu.cn

${ }^{b}$ School of Mathematical Science, Tianjin Normal University, Tianjin 300387, China 'Tianjin University of Science and Technology, Tianjin 300222, China process. $^{7}$ In 1924, J. P. Hooper firstly proposed a patent called "centrifugal spinneret": due to the limitations of the experimental conditions at that time, the fibers produced were relatively large in diameter and unstable in the production process. It was not until the middle and late 20th century that people gradually began to pay attention to it, which is still being developed and perfected. Although it is still in its infancy in domestic industrial production, it has broad development prospects due to its simple and flexible equipment and high production efficiency.

Wound healing is a complicated biological process involving numerous physiological factors. To accelerate the healing process, wound dressings have been used since the ancient times. ${ }^{8}$ Wound dressings, which were used to treat wound exudate and protect wounds from bacterial and dust particles, have developed rapidly and are gradually showing a trend of functionalization and diversification in recent years. ${ }^{9,10}$ The drug-loaded fibers obtained by centrifugal spinning have many advantages in wound dressing. For example, nanofiber can increase the effective surface area of the drug, and the high porosity is favorable for cell adhesion and proliferation, and the gas permeability and moisture permeability are good for cell growth. Thus, the formed porous fiber scaffold provides a good environment for wound repair and healing, providing a good environment for wound healing, various anti-inflammatory and analgesic drugs are generally added to the dressing at the same time. ${ }^{11}$ Due to the break 
release of the drug in the ordinary release system, the concentration of the drug is too high and repeated drug administrations, which brings great harm to the living organism. Therefore, the drug can be loaded into the fibers to achieve the purpose of reducing the toxic side effects and repeated administration of the excessive drug to the human body. ${ }^{12-14}$

Among all different types of synthetic polymers, poly(lactic acid) (PLA) has been widely used in recent years because it is an environmentally benign material for potential application prospect as a substitute for medical dressings. ${ }^{15}$ This is owed greatly to the polymer's favorable biocompatibility and to its safe degradation products. Once coming in contact with biological media, the polymer begins breaking down, usually by hydrolysis, into lactic acid or to carbon dioxide and water. Although PLA represents good properties, low hydrophilicity undermines cell attachment, proliferation, and migration. ${ }^{\mathbf{1 6}}$ To deal with low biocompatibility of PLA, gelatin (GE) can be blended, which is a widely used protein in various fields of biomedical applications due to its hydrophilicity, biocompatibility, biodegradability, low cost and numerous available active groups for attaching targeting molecules. ${ }^{17,18}$

Ciprofloxacin hydrochloride (CPF) is one of the outstanding representatives of quinolone antibacterial and a chemically synthesized broad-spectrum antibacterial drug developed by Bayer Pharmaceuticals in Germany in 1983. CPF mainly exerts rapid bactericidal action by inhibiting bacterial DNA gyrase, and has strong antibacterial activity against bacteria such as Gram-negative bacteria, Gram-positive bacteria and Mycoplasma. ${ }^{19}$ In addition, as CPF has few toxicity and low resistance to drug resistance, it is widely used in the treatment of infectious diseases in animals and humans, ${ }^{20-22}$ and has a large sales ratio in the international market, even has developed rapidly in domestic production in recent years.

At present, there are only few reports on the preparation of PLA nanofibers by centrifugal spinning. In this paper, PLA was blended with GE and CPF to improve the hydrophilicity and antibacterial properties of the PLA nanofibers. According to a large number of papers, this paper is the first time to successfully prepare PLA/GE/CPF nanofibers using a self-made centrifugal spinning machine and the obtained fibers have potential applications in the medical field.

\section{Experimental section}

\subsection{Materials}

PLA $\left(M_{\mathrm{w}} 150000\right)$ pellets and GE powder were purchased from Sigma-Aldrich. CPF drug was purchased from Sinopharm Chemical Reagent Co., Ltd (Shanghai, China). 1,1,1,3,3,3Hexafluoro-2-propanol and sodium hydroxide $(\mathrm{NaOH})$ was purchased from Tianjin Kairuisi Fine Chemical Co. Ltd (Tianjin, China). Staphylococcus aureus (S. aureus) and Escherichia coli (E. coli) were purchased from Shanghai Lu Microbiology Technology Co., Ltd (Shanghai, China). All the chemicals were analytical grade and were used without further purification.

\subsection{Preparation of PLA/GE/CPF nanofibers}

The 5 parts PLA/GE ( $85: 15 / \mathrm{w}: \mathrm{w})$ solution with a concentration of $6 \%$ was prepared by mixing the materials in 1,1,1,3,3,3hexafluoro-2-propanol at stirring on a magnetic stirrer for 6 hours, then the different amounts of CPF (CPF: PLA/GE $=0 \%$, $6 \%, 8 \%, 10 \%, 12 \%)$ drugs were added to the above solution until a homogeneous dispersion was obtained and left it for several hours to let bubbles disappear. In the experiment, the polymer solution was placed in two $5 \mathrm{ml}$ plastic syringes with a needle diameter of $0.11 \mathrm{~mm}$, which were attached to the rotating blades by nylon cable ties. The solution was ejected under the centrifugal force generated by a rotation speed of $5500 \mathrm{rpm}$ and the jets were stretched under centrifugal force, accompanied by the evaporation of solvent before being collected. The distance between the spinneret and the receiving device was set $15 \mathrm{~cm}$.

All spinning experiments were performed in ambient room temperature. Fig. 1 showed the schematic diagram of the centrifugal spinning apparatus, which was consisted of two centrifugal chambers with spinneret, a Direct Current (DC) motor and a series of rod collectors.

\subsection{Characterization methods}

2.3.1. Scanning electron microscopy (SEM). The surface morphologies and diameters of the PLA/GE/CPF nanofibers were investigated by desktop scanning electron microscope (SEM) (FlexSEM 1000, Japan Hitachi Nake High-Tech Enterprise, Japan). The diameters of the nanofibers were measured using the image analysis software Image $\mathrm{J}$ on a minimum of 50 individual fibers per specimen, with two specimens per sample and with multiple readings per individual fiber to account for potential variability along the axis of fibers.

2.3.2. Fourier transform infrared (FTIR). The presence of CPF in the nanofibers were identified using Fourier Transform Infrared Spectroscopy (FTIR) (Nicolet iS50, Thermo Fisher Scientific Co., Ltd., China). The FTIR spectra were collected with a resolution of $4 \mathrm{~cm}^{-1}$ at $4000-400 \mathrm{~cm}^{-1}$.

2.3.3. SEM coupled with energy dispersive spectroscopy (EDS). The morphology of surfaces of nanofibers was studied by using scanning electron microscopy (SEM). It is a technique of morphological analysis based on the principle of electronmatter interactions. To allow surface conduction, the nanofibers were metalized by gold-palladium layer (a few $\mu \mathrm{m}$ of

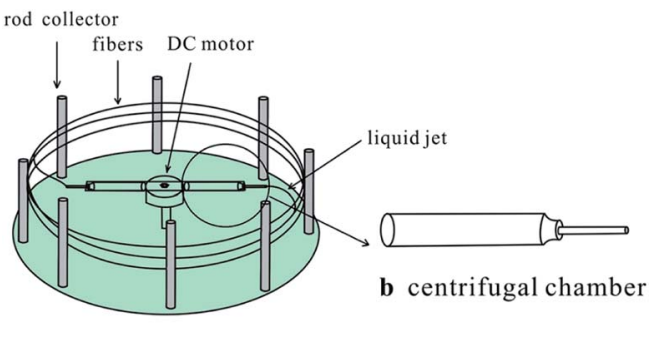

a centrifugal spinning device

Fig. 1 Schematic diagram of the centrifugal spinning device. ${ }^{4}$ 
thickness) before being introduced into the analysis room. Semi quantitative chemical analysis on nanofibers surfaces was performed by energy dispersive spectroscopy (EDS) in Model 550i.

2.3.4. X-ray diffraction (XRD). The crystallinity of the CPF, PLA and GE materials and nanofibers were investigated using $\mathrm{X}$ ray diffraction (XRD) (D8 Discover with GADDS, Bruker AXS Co., Ltd, USA). The XRD was performed in the range of $(2 \theta) 10-50^{\circ}$ at 2 grad $\min ^{-1}$ scanning rate at room temperature.

2.3.5. Thermogravimetric (TG). The thermal degradation was determined using thermogravimetric analyzer (TGA) (STA449F3, Germany Netzsch). The heating was performed from 25 to $600{ }^{\circ} \mathrm{C}$ at a heating rate of $10^{\circ} \mathrm{C} \mathrm{min}{ }^{-1}$ in nitrogen atmosphere.

2.3.6. Contact angle measurements. The static contact angles of all the samples were measured with deionized water as a fluid, using optical contact angle measuring instrument (DSA 100, Beijing Shangdetong Technology Co., Ltd., China). Five measurements were performed on different positions on the samples.

2.3.7. Antimicrobial test. Round samples $(R=14 \mathrm{~mm})$ were placed in Petri dishes containing solid agar medium seeded with Staphylococcus aureus or Escherichia coli. Each plate was incubated at $37{ }^{\circ} \mathrm{C}$ for 24 hours. Inhibition zone was measured perpendicularly from the center of the sample to the inner periphery of the bacterial colonies around, after $24 \mathrm{~h}$ of incubation.

2.3.8. In vitro drug release study. The centrifugally spun nanofibers were cut, accurately weighed and incubated in $10 \mathrm{~mL}$ of phosphate buffer saline (PBS) $(\mathrm{pH} 7.4)$ at $37{ }^{\circ} \mathrm{C}$ with mild shaking $(110 \mathrm{rpm})$ in a air bath. At predetermined time points of $1,5,12,24,48,72,96,120,144 \mathrm{~h}$ and $168 \mathrm{~h}$, eluents of $1 \mathrm{ml}$ for each sample was withdrawn and assayed using a UV-Vis spectrophotometer at a wavelength of $276 \mathrm{~nm}$. An equal volume of fresh buffer was replaced after each withdrawal. The collected eluents were analyzed by UV-Vis spectrophotometry to obtain cumulative release amount profiles and cumulative release percentage profiles. The release study was performed in triplicate and the mean was calculated.

\section{Results and discussion}

\subsection{SEM image analysis}

An important factor for the successful centrifugal spinning of any synthetic or natural polymers consists in the preparation of solutions with adequate concentrations. At low concentration, the solution has low viscosity and less entanglement between molecular chains, which influences the morphology of fibers, even forms droplets or beads rather than fibers below a certain concentration. At high concentrations, centrifugal spinning is prohibited by an inability to control and maintain the flow of a polymer solution to the nozzle. In this study, nanofibers loaded with different CPF drugs concentrations were successfully prepared by centrifugal spinning, and SEM images of centrifugally spun PLA/GE/CPF nanofiber samples are shown in Fig. 2. With the increasing of the CPF concentration, the diameters of the nanofibers were $513 \mathrm{~nm}, 565 \mathrm{~nm}, 584 \mathrm{~nm}$, $594 \mathrm{~nm}$ and $622 \mathrm{~nm}$ when the CPF concentration were $0 \%, 6 \%$,
$8 \%, 10 \%$ and $12 \%$, respectively. This is mainly because the solution viscosity and chain entanglement were gradually enhanced, resulting in an increase in diameter and in surface smoothness of nanofibers. In addition, the smooth surface of the nanofibers is a great advantage for the wound dressing, which can hardly cause secondary injury to the wound.

\subsection{FTIR and EDS analysis}

To verify the successful loading of CPF, the CPF drugs and nanofibers with different CPF concentrations were characterized by FTIR testing, as shown in Fig. 3. The absorption peaks of the infrared spectra of PLA/GE nanofibers curves were observed at 1184 and $1087 \mathrm{~cm}^{-1}$, which were caused by C-O-C stretching vibration, the absorption peaks at $1752 \mathrm{~cm}^{-1}$ was caused by $\mathrm{C}=\mathrm{O}$ stretching vibration; the absorption peaks at 1654 and $1540 \mathrm{~cm}^{-1}$ were respectively corresponding to the amide I band $\mathrm{C}=\mathrm{O}$ stretching vibration absorption peak and amide II band $\mathrm{N}-\mathrm{H}$ symmetric deformation absorption peak. ${ }^{23}$ The absorption peaks of CPF curves at $1626 \mathrm{~cm}^{-1}$ was the characteristic vibration absorption peak of $\mathrm{C}=\mathrm{O}$ groups. ${ }^{24,25}$ For the CPF-loaded nanofibers, besides the characteristic absorption peaks of the $\mathrm{CPF}$, peaks belonging to were also observed in the FTIR spectrum of CPF-loaded nanofibers. In addition, with the increase of the CPF content, the characteristic vibration absorption peak attributed to the CPF gradually strengthens.

Although the absorption peak of the CPF-exclusive functional group was shown in the FTIR spectrum, the absorption peak was not significant because the amount of CPF added was too small. To further demonstrate that CPF was successfully loaded on the nanofibers, the EDS element mapping was employed, which revealed the presence of $\mathrm{F}$ that confirm the CPF was successfully loaded on the nanofibers, as shown in Fig. 4.

\subsection{XRD analysis}

Fig. 5 shows the XRD patterns of the CPF drugs and PLA/GE/ $\mathrm{CPF}$ nanofibers at different $\mathrm{CPF}$ concentrations. According to the curve, the PLA/GE nanofibers before drug loading showed half-diffraction peaks at $16.6^{\circ}, 27^{\circ}$ and $28.9^{\circ}$ due to insufficient crystallization of the nanofibers. The $2 \theta$ value of the peaks in $\mathrm{CPF}$ were $8.3^{\circ}, 9.1^{\circ}$ and $19.3^{\circ}$, etc. However, the characteristic peaks belonging to CPF disappear after loading the nanofibers, and the characteristic peaks belonging to PLA/GE were weakened, which indicated that the small molecular weight CPF were existed in amorphous state in nanofibers and hinders the arrangement of macromolecular chains in the nanofibers.

\subsection{TG analysis}

The thermal properties of the PLA/GE/CPF nanofibers prepared by centrifugal spinning were summarized in Fig. 6 . The affect of the CPF concentrations on the thermal stability of the PLA/GE/ CPF nanofibers was evaluated by TGA and two different stages of the weight loss were detected on the obtained thermograms. In the first stage, the observed weight loss of the nanofibers can be attributed to the degradation of GE and the evaporation of the adsorbed water. The second stage of mass loss is the 

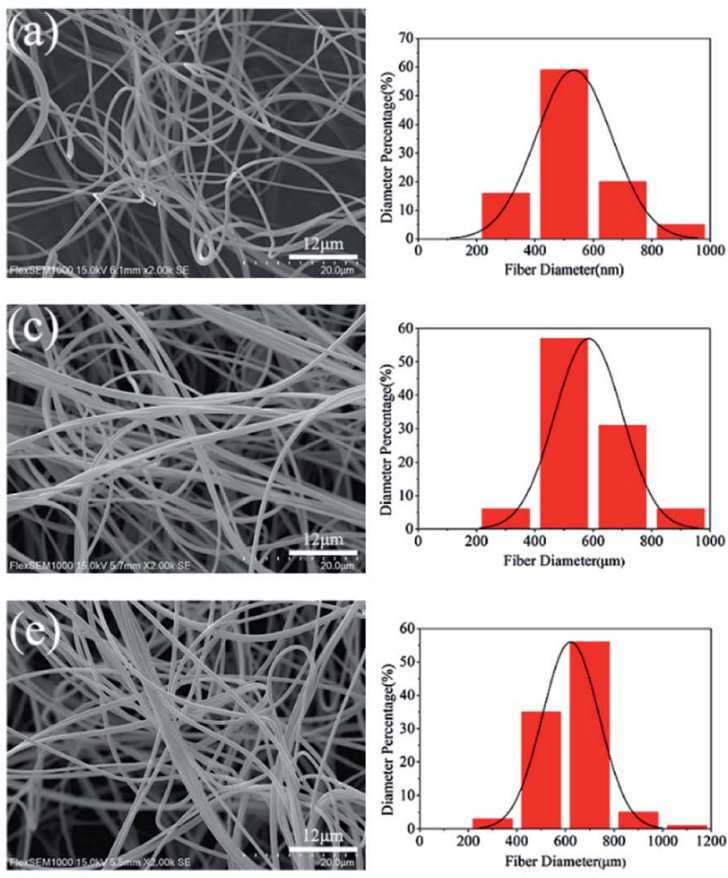
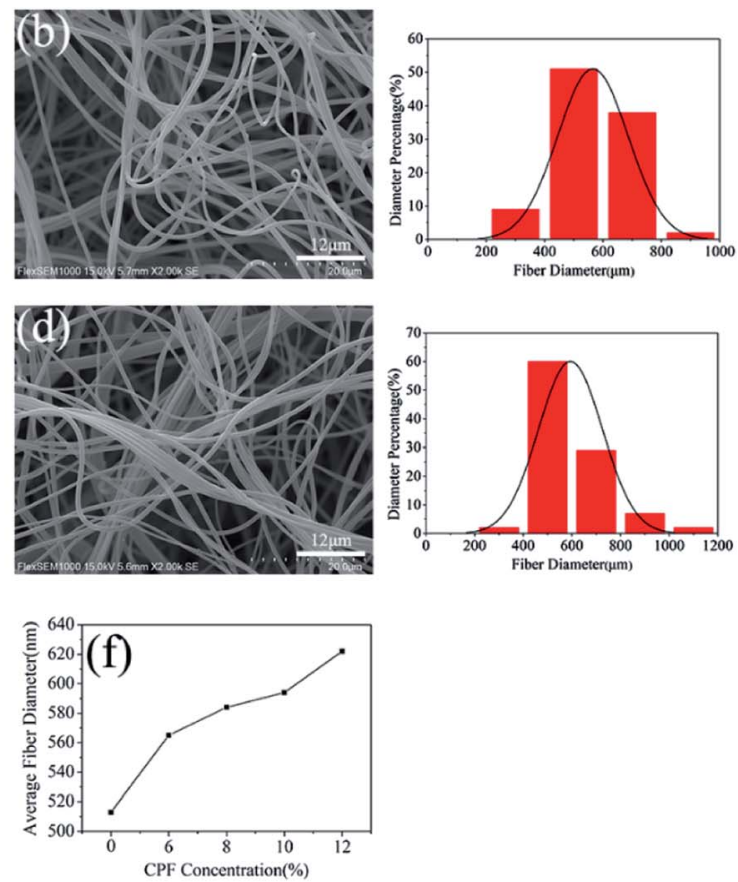

Fig. 2 SEM images of PLA/GE/CPF nanofibers and diameter distributions at rotational speed of $5500 \mathrm{rpm}$, collecting distance of $15 \mathrm{~cm}$, spinneret diameter of $0.11 \mathrm{~mm}$ and CPF concentration of (a) $0 \%$, (b) $6 \%$, (c) $8 \%$, (d) $10 \%$, (e) $12 \%$. (f) The average diameter of nanofibers prepared with different CPF concentration.

degradation of PLA, which can be seen at this stage the thermal characteristics shift to the lower temperatures and the degradation becomes faster with an increase in the CPF concentrations. This phenomenon may be related to the crystallinity of the nanofiber. However, the observed effect of the CPF concentrations on the thermal stability of the nanofibers is not significant because the change in the decomposition temperature is relatively small.

\subsection{Hydrophilicity of PLA/GE/CPF nanofibers}

The hydrophilicity of PLA/GE nanofibers was shown in Fig. $7\left(a_{1}\right)$. The average contact angle of PLA/GE nanofibers was $81^{\circ}$ because the hydrophilicity of pure PLA fibers was improved by adding GE. The average contact angles of nanofibers were $75^{\circ}, 72^{\circ}, 68^{\circ}$ and $47^{\circ}$ when CPF concentration was $6 \%, 8 \%, 10 \%$ and $12 \%$, respectively. The contact angles of the nanofibers loading the drug decreased significantly as the concentration of
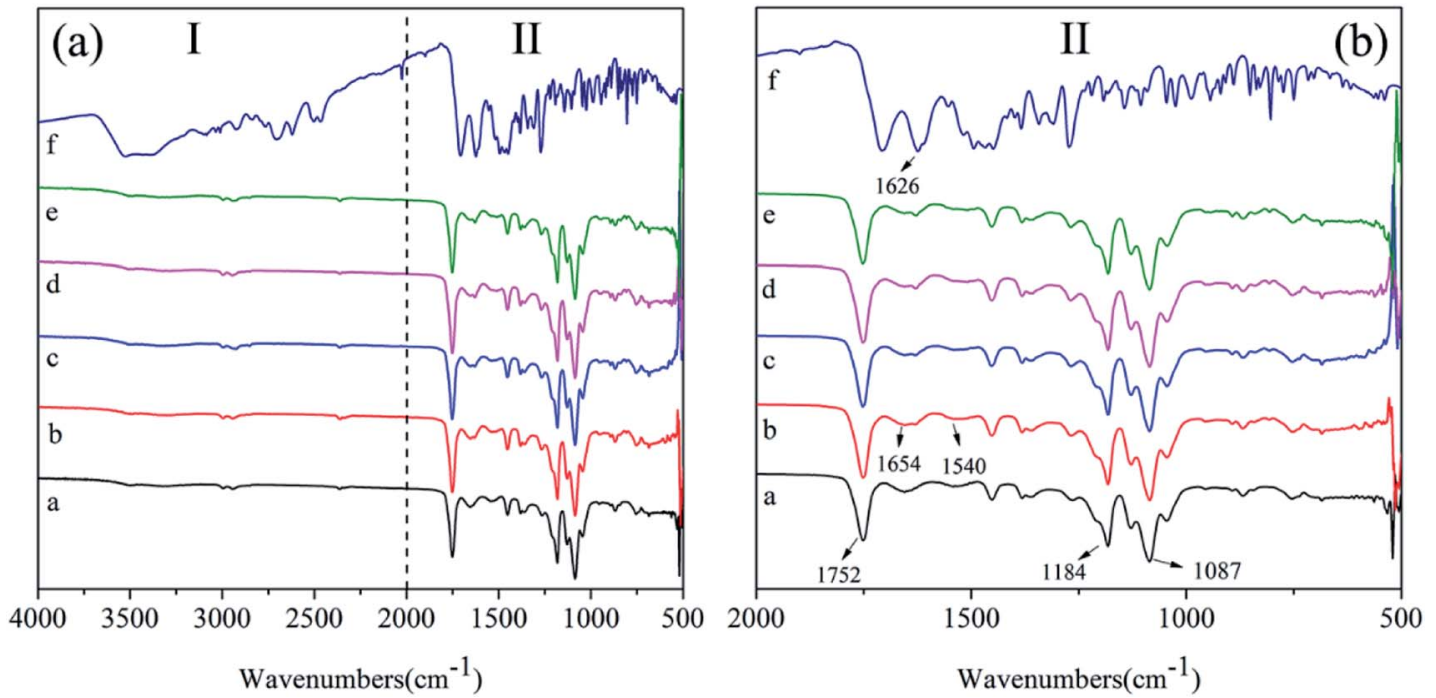

Fig. 3 FTIR curves of PLA/GE/CPF nanofibers with CPF concentration of (a) $0 \%$, (b) $6 \%$, (c) $8 \%$, (d) $10 \%$, (e) $12 \%$, and (f) CPF drugs. Spectra (I) from $4000 \mathrm{~cm}^{-1}$ to $2000 \mathrm{~cm}^{-1}$ and (II) from $2000 \mathrm{~cm}^{-1}$ to $500 \mathrm{~cm}^{-1}$. 

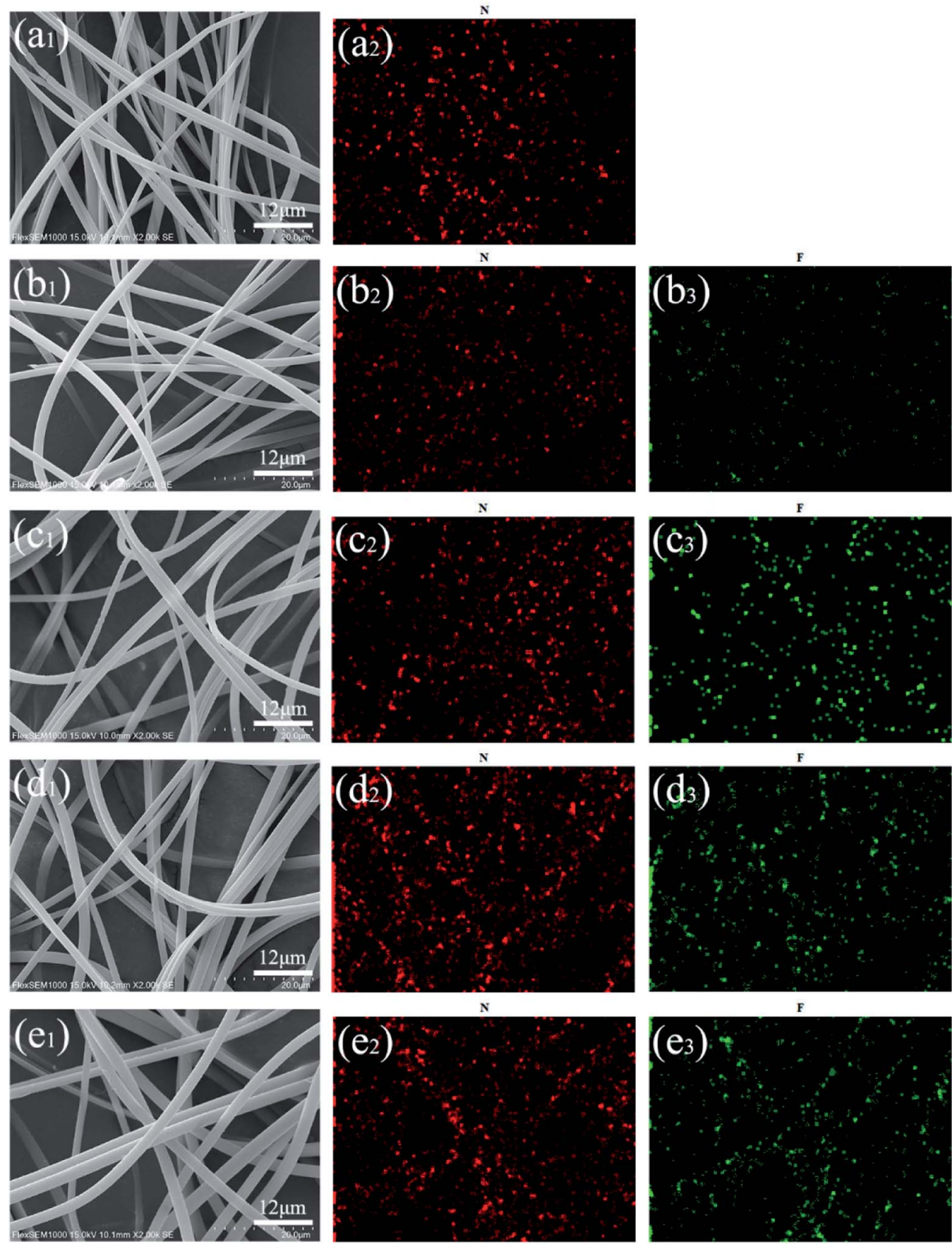

Fig. 4 EDS images of PLA/GE/CPF nanofibers at CPF concentration of $\left(a_{1}\right) 0 \%,\left(b_{1}\right) 6 \%,\left(c_{1}\right) 8 \%,\left(d_{1}\right) 10 \%,\left(e_{1}\right) 12 \%$. EDS images of $N$ element in the PLA/GE/CPF nanofibers at CPF concentration of $\left(a_{2}\right) 0 \%,\left(b_{2}\right) 6 \%,\left(c_{2}\right) 8 \%,\left(d_{2}\right) 10 \%,\left(e_{2}\right) 12 \%$. EDS images of $F$ element in the PLA/GE/CPF nanofibers at CPF concentration of $\left(b_{3}\right) 6 \%,\left(c_{3}\right) 8 \%,\left(d_{3}\right) 10 \%,\left(e_{3}\right) 12 \%$.

CPF increased, which is because the loaded CPF is hydrophilic. The application of hydrophilic nanofibers to wound dressings not only facilitates cell adhesion, but also facilitates cell proliferation and differentiation, which provides a good environment for wound healing.

\subsection{Antibacterial performance analysis}

The antibacterial test method in this paper was the standard agar diffusion method. The antibacterial properties of PLA/GE/ CPF nanofibers in terms of zone of inhibition against Gram- positive bacteria (E. coli) and Gram-negative bacteria ( $S$. aureus) were shown in Fig. 8(a) and (b). The fibers before loading CPF drugs had no antimicrobial activities against the two bacteria tested after $24 \mathrm{~h}$ of incubation, while the fibers after loading drugs had significant inhibition zones for both bacteria. The inhibition zones of fibers to $E$. coli were $0 \mathrm{~mm}, 24$ $\mathrm{mm}, 28 \mathrm{~mm}, 32 \mathrm{~mm}$ and $36 \mathrm{~mm}$, and to $S$. aureus were $0 \mathrm{~mm}, 36$ $\mathrm{mm}, 38 \mathrm{~mm}, 40 \mathrm{~mm}$ and $44 \mathrm{~mm}$ when CPF concentration was $0 \%, 6 \%, 8 \%, 10 \%$ and $12 \%$, respectively. The above experimental results showed that the diameter of the inhibition zones 


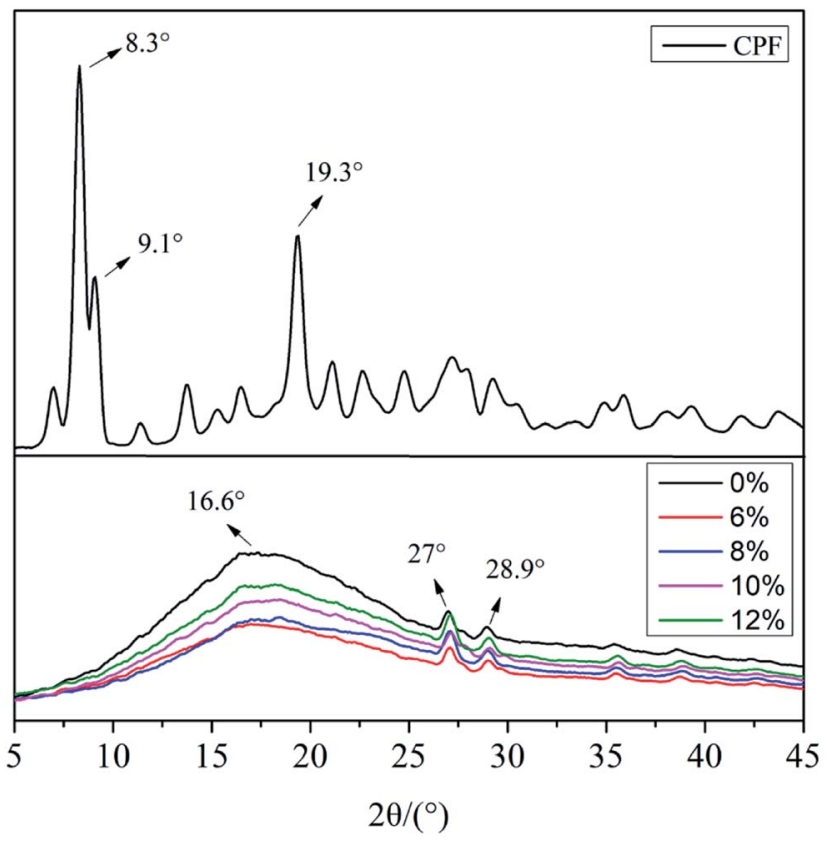

Fig. 5 XRD curves of CPF drugs and PLA/GE/CPF nanofibers with CPF concentration of $0 \%, 6 \%, 8 \%, 10 \%$ and $12 \%$.

increased with the increase of CPF concentration, and the inhibitory effect of CPF drug on $S$. aureus was better than that of E. coli.

\subsection{In vitro drug release analysis}

Fig. 8(c) and (d) exhibits drug release amount profiles and percentage profiles of samples in various time intervals. The release behaviors of CPF drugs from the nanofibers in vitro were evaluated in PBS ( $\mathrm{pH}$ 7.4) to simulate the physiological conditions of the human body. As shown in the figures, the release curve value of the nanofibers without the CPF drug was always 0 while the release profiles of all the nanofibers containing the CPF drug showed a burst release at the initial stage, and about $30 \%$ of CPF drugs were released in $1 \mathrm{~h}$ and exhibited a relatively slow release property after the initial burst time. The relatively
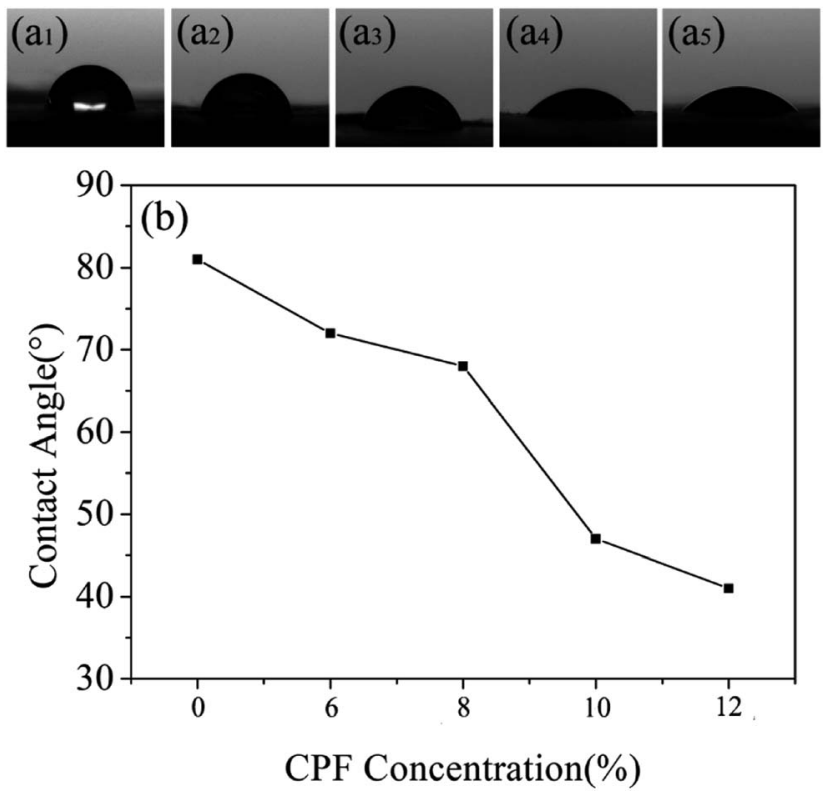

Fig. 7 The contact angles of nanofibers with CPF concentrations of $\left(a_{1}\right) 0 \%,\left(a_{2}\right) 6 \%,\left(a_{3}\right) 8 \%,\left(a_{4}\right) 10 \%$ and $\left(a_{5}\right) 12 \%$. (b) The contact angle line diagram of nanofibers.

fast release in the early stage was likely attributed to the release of CPF drugs adsorbed on the surface of the nanofibers. The dissolution of these drugs opened up several pores through which the release medium could penetrate the interior of the nanofibers and dissolve the drugs reserved in the pores, thus releasing them. The mass transfer process between the release medium and the drugs within the apertures was hindered by the PLA polymer chains, and therefore sustained drug release was observed. In addition, it can be seen from Fig. 8(c) that during the entire release process, the more drugs were released by the fibers as the CPF concentration increased during the same time. In particular, when the CPF concentration was $10 \%$ and $12 \%$, the release of fibers reached $87 \%$ in $144 \mathrm{~h}$. This is mainly due to the increase of CPF content both within and on the surface of the nanofibers. However, the release percentages
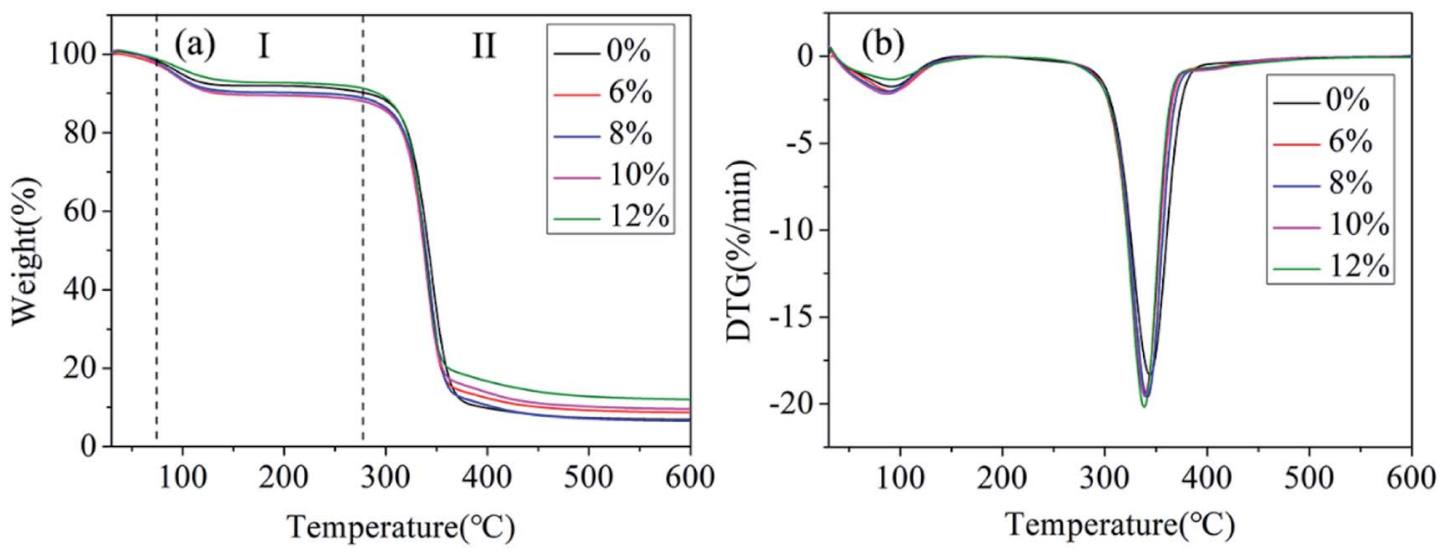

Fig. 6 (a) TG and (b) DTG curves of PLA/GE/CPF nanofibers with CPF concentration of $0 \%, 6 \%, 8 \%, 10 \%$ and $12 \%$. 

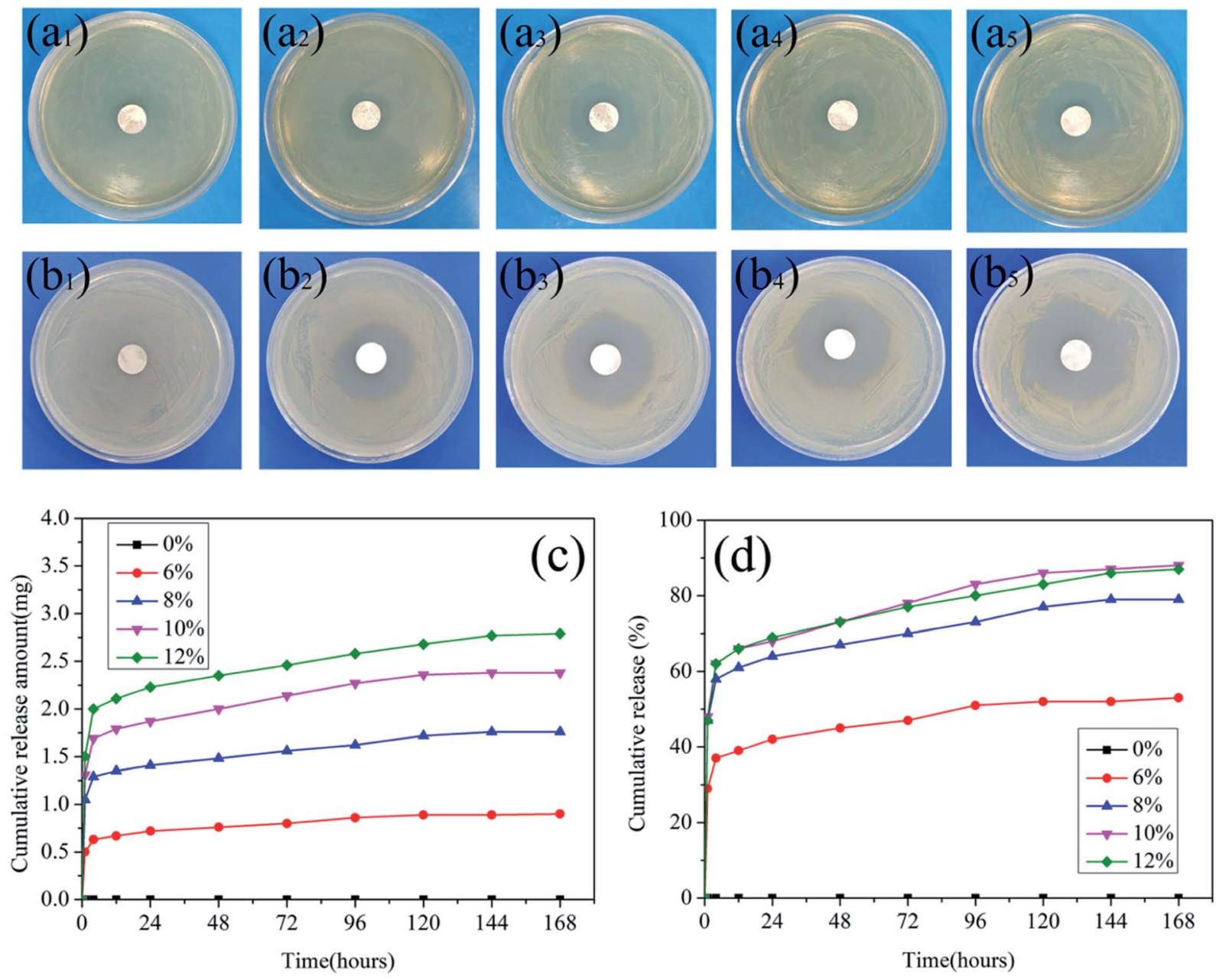

Fig. 8 The antibacterial effect of different concentrations of CPF $\left(\left(a_{1}\right) 0 \%,\left(a_{2}\right) 6 \%,\left(a_{3}\right) 8 \%,\left(a_{4}\right) 10 \%,\left(a_{5}\right) 12 \%\right)$ on $E$. coli, and the antibacterial effect of different concentrations of CPF $\left(\left(b_{1}\right) 0 \%,\left(b_{2}\right) 6 \%\right.$, $\left.\left(b_{3}\right) 8 \%,\left(b_{4}\right) 10 \%,\left(b_{5}\right) 12 \%\right)$ on $S$. aureus. (c) In vitro drug release amount profiles from nanofibers with different CPF concentrations. (d) In vitro drug release percentage profiles from nanofibers with different CPF concentrations.

of nanofibers with $10 \% \mathrm{CPF}$ concentration is higher than that of nanofibers with $12 \%$ CPF concentration as shown in the Fig. 8(d). This is mainly because the diameter of the nanofiber with $12 \% \mathrm{CPF}$ concentration is larger than that of the nanofiber with $10 \% \mathrm{CPF}$ concentration, and the drug release within the fiber takes a longer time. In general, the release amount of nanofibers with $12 \% \mathrm{CPF}$ concentration is larger than that of the nanofiber with $10 \% \mathrm{CPF}$ concentration.

\section{Conclusion}

In this paper, a design method for improving the antibacterial properties of PLA/GE nanofibers was prepared by centrifugal spinning technology. In summary, nanofibers loaded with different CPF drugs concentrations were successfully obtained by centrifugal spinning. The results indicated that the diameter of nanofibers increased as the concentration of CPF increased, and the surface of the nanofibers were smooth. Fourier transform infrared spectroscopy test results showed that CPF drugs were successfully loaded on the nanofibers. The X-ray diffraction analysis proved that CPF drugs were amorphous in the nanofibers and hinders the arrangement of macromolecular chains in the nanofibers. The results obtained from thermogravimetric indicated that the effect of the concentration of CPF on the thermal stability of the nanofibers was not significant. The water contact angle of the nanofibers decreased as the concentration of the drug loaded increased, which showed that the hydrophilicity of the nanofibers was significantly improved. Antibacterial tests showed the antibacterial effect of the fiber was better as the CPF concentration increased. In addition, at the same CPF drugs concentration, the inhibition zone of the fibers against $S$. aureus was larger than that of $E$. coli, indicating that the drug is sensitive to Staphylococcus aureus. In vitro drug release study indicated that all the nanofibers containing the CPF drugs had a burst release at the initial stage, and about $30 \%$ of CPF drugs were released in $1 \mathrm{~h}$ and exhibited a relatively slow release property after the initial burst time. In particular, when the CPF concentration was $10 \%$ and $12 \%$, the release of fibers reached $87 \%$ in $144 \mathrm{~h}$, which played a sustained release effect. All the above results proved that PLA/ $\mathrm{GE} / \mathrm{CPF}$ nanofibers prepared by centrifugal spinning technology has potential prospects for dressings. 


\section{Conflicts of interest}

There are no conflicts to declare.

\section{Acknowledgements}

This study was supported by the Tianjin Natural Science Foundation (grant no. 18JCQNJC71900).

\section{References}

1 A. E. Erickson, D. F. Edmondson, C. Chang, D. Wood, A. Gong, S. L. Levengood and M. Zhang, High-throughput and high-yield fabrication of uniaxially-aligned chitosanbased nanofibers by centrifugal electrospinning, Carbohydr. Polym., 2015, 134, 467-474.

2 R. Lv, Y. Zhu, H. Liu, B. Na, Y. Huang and X. Xie, Poly(vinylidene fluoride)/poly(acrylonitrile) blend fibrous membranes by centrifugal spinning for high-performance lithium ion battery separators, J. Appl. Polym. Sci., 2017, 134(8), 44515.

3 R. Nava, L. Cremar, V. Agubra, J. Sanchez, M. Alcoutlabi and K. Lozano, Centrifugal spinning: an alternative for large scale production of silicon-carbon composite nanofibers for lithium ion battery anodes, ACS Appl. Mater. Interfaces, 2016, 8(43), 29365-29372.

4 L. Xia, J. Ju, W. Xu, C. Ding and B. Cheng, Preparation and characterization of hollow $\mathrm{Fe}_{2} \mathrm{O}_{3}$ ultra-fine fibers by centrifugal spinning, Mater. Des., 2016, 96, 439-445.

5 W. Xu, P. Xi, L. Xia, B. Cheng, X. Zhou and Y. Liang, Hollow carbon microfibres fabricated using coaxial centrifugal spinning, Micro Nano Lett., 2016, 11(2), 74-76.

6 P. Mellado, H. A. McIlwee, M. R. Badrossamay, J. A. Goss, L. Mahadevan and K. K. Parker, A simple model for nanofiber formation by rotary jet-spinning, Appl. Phys. Lett., 2011, 99(20), 203107.

7 H. Zhao, X. Min, X. Wu, H. Wang, J. Liu, Z. Zhang, Z. Huang, Y. Liu and M. Fang, Microstructure and electrochemical properties of polyacrylonitrile-based carbon micro- and nanofibers fabricated by centrifugal spinning, Chem. Phys. Lett., 2017, 684, 14-19.

8 P. Zahedi, I. Rezaeian, S. O. Ranaei-Siadat, S. H. Jafari and P. Supaphol, A review on wound dressings with an emphasis on electrospun nanofibrous polymeric bandages, Polym. Adv. Technol., 2009, 21(2), 77-95.

9 A. Abdal-Hay, F. A. Sheikh and J. K. Lim, Air jet spinning of hydroxyapatite/poly(lactic acid) hybrid nanocomposite membrane mats for bone tissue engineering, Colloids Surf., $B, 2013,102,635-643$.

10 F. Amini, D. Semnani, S. Karbasi and S. N. Banitaba, A novel bilayer drug-loaded wound dressing of PVDF and PHB/ chitosan nanofibers applicable for post-surgical ulcers, Int. J. Polym. Mater. Polym. Biomater., 2018, 68(13), 772-777.

11 Y. Tang, X. Lan, C. Liang, Z. Zhong, R. Xie, Y. Zhou, X. Miao, H. Wang and W. Wang, Honey loaded alginate/PVA nanofibrous membrane as potential bioactive wound dressing, Carbohydr. Polym., 2019, 219, 113-120.
12 J. G. Lundin, C. L. McGann, G. C. Daniels, B. C. Streifel and J. H. Wynne, Hemostatic kaolin-polyurethane foam composites for multifunctional wound dressing applications, Mater. Sci. Eng. C, 2017, 79, 702-709.

13 M. S. Refat, K. M. Elsabawy, A. Alhadhrami, A. S. A. Almalki, M. Y. El-Sayed and R. F. Hassan, Development of medical drugs: synthesis and in vitro bio-evaluations of nanomedicinal zinc-penicillins polymeric hydrogel membranes for wound skin dressing by new chemical technology, J. Mol. Liq., 2018, 255, 462-470.

14 B. K. Huh, B. H. Kim, S. N. Kim, C. G. Park, S. H. Lee, K. R. Kim, C. Y. Heo and Y. B. Choy, Surgical suture braided with a diclofenac-loaded strand of poly(lactic-coglycolic acid) for local, sustained pain mitigation, Mater. Sci. Eng. C, 2017, 79, 209-215.

15 E. T. H. Vink, K. R. Rabago, D. A. Glassner and P. R. Gruber, Applications of life cycle assessment to NatureWorks ${ }^{\mathrm{TM}}$ polylactide (PLA) production, Polym. Degrad. Stab., 2003, 80(3), 403-419.

16 K. T. Shalumon, S. Deepthi, M. S. Anupama, S. V. Nair, R. Jayakumar and K. P. Chennazhi, Fabrication of poly(Llactic acid)/gelatin composite tubular scaffolds for vascular tissue engineering, Int. J. Biol. Macromol., 2015, 72, 1048-1055.

17 N. Sahoo, R. K. Sahoo, N. Biswas, A. Guha and K. Kuotsu, Recent advancement of gelatin nanoparticles in drug and vaccine delivery, Int. J. Biol. Macromol., 2015, 81, 317-331.

18 O. Elzoghby, Gelatin-based nanoparticles as drug and gene delivery systems: reviewing three decades of research, $J$. Controlled Release, 2013, 172(3), 1075-1091.

19 H. Li, G. R. Williams, J. Wu, H. Wang, X. Sun and L. M. Zhu, Poly( $N$-isopropylacrylamide)/poly(L-lactic acid-co-varepsiloncaprolactone) fibers loaded with ciprofloxacin as wound dressing materials, Mater. Sci. Eng. C, 2017, 79, 245-254.

20 M. F. Sanchez, S. A. Breda, E. A. Soria, L. I. Tartara, R. H. Manzo and M. E. Olivera, Ciprofloxacin-lidocainebased hydrogel: development, characterization, and in vivo evaluation in a second-degree burn model, Drug Delivery Transl. Res., 2018, 8(5), 1000-1013.

21 Z. Rezvani and M. Shahbaei, Bionanocomposites based on alginate and chitosan/layered double hydroxide with ciprofloxacin drug: investigation of structure and controlled release properties, Polym. Compos., 2015, 36(10), 1819-1825.

22 A. Doustgani, Doxorubicin release from optimized electrospun polylactic acid nanofibers, J. Ind. Text., 2016, 47(1), 71-88.

23 L. Ji, M. Gong, W. Qiao, W. Zhang, Q. Liu, R. E. Dunham and J. Gu, A gelatin/PLA-b-PEG film of excellent gas barrier and mechanical properties, J. Polym. Res., 2018, 25(10), 210-222.

24 H. Li, G. R. Williams, J. Wu, Y. Lv, X. Sun, H. Wu and L. M. Zhu, Thermosensitive nanofibers loaded with ciprofloxacin as antibacterial wound dressing materials, Int. J. Pharm., 2017, 517(1-2), 135-147.

25 Q. Sang, H. Li, G. Williams, H. Wu and L. M. Zhu, Core-shell poly(lactide-co-e-caprolactone)-gelatin fiber scaffolds as $\mathrm{pH}$ sensitive drug delivery systems, J. Biomater. Appl., 2018, 32(8), 1105-1118. 